\title{
Chronic cerebrospinal venous insufficiency: A Kuhnian paradigm shift or another fad?
}

\author{
Robert J. Fox MD
}

See related research article by Laupacis and colleagues at www.cmaj.ca/lookup/doi/10.1503/cmaj.111074 and synopsis on page 1847 .

Competing interests:

Robert Fox has served as a consultant for Avanir, Biogen Idec, Genentech and Novartis; has served on clinical trial advisory committees for Biogen

Idec; has received research support from the National Multiple Sclerosis Society (RG 4091A3/1; RG

4103A4/2; RC 1004-A-5), which includes funding to study chronic cerebrospinal venous insufficiency; and serves on the editorial boards of Neurology and Multiple Sclerosis Journal.

This article was solicited and has not been peer reviewed.

Correspondence to: Dr. Robert J. Fox, foxr@ccf.org

CMAJ 2011. DOI:10.1503 /cmaj.111506
$\mathrm{M}$ ultiple sclerosis is a chronic demyelinating disorder affecting the brain, spinal cord and optic nerves. Although the cause of multiple sclerosis is unknown, pathologic studies suggest that the patient's immune system inappropriately attacks the myelin sheath covering axons. All currently approved diseasemodifying treatments for multiple sclerosis target the immune system in one way or another.

There have been many theories as to the cause of multiple sclerosis, including toxins, infectious agents and primary neurodegeneration. Perhaps one of the oldest theories relates to vascular abnormalities, first described by 19th century pathologist Jean Cruveilhier, ${ }^{1}$ and subsequently by others including Jean-Martin Charcot. $^{2}$ The vascular theory re-emerged several times during the 20th century: with trials of anticoagulant drugs in the 1940s, with pathologic and venographic studies that hypothesized injury caused by venous backflow in the 1960s and 1970s, and with trials using hyperbaric oxygen in the 1970s. With advances in immunology and immunotherapies, the vascular theory of multiple sclerosis all but disappeared at the close of the 20th century.

Starting around 2007, a series of publications led by Italian vascular surgeon Paolo Zamboni brought renewed attention to the vascular theory of multiple sclerosis. ${ }^{3}$ Zamboni and colleagues hypothesized that multiple sclerosis was caused by impaired venous outflow in the neck, with secondary injury to the brain and spinal cord from venous backflow. They used ultrasonographic imaging of the internal jugular, vertebral and intracranial veins to define what they termed "chronic cerebrospinal venous insufficiency." The criteria for this phenomenon, as seen using ultrasonographic imaging, focus on venous reflux, stenosis and altered postural control of the jugular and intracranial veins (Appendix 1, available at www.cmaj.ca/lookup/suppl/doi:10 $.1503 / \mathrm{cmaj} .111506 /$ /DC1). Fulfillment of two or more of these criteria equates to a diagnosis of chronic cerebrospinal venous insufficiency, with a surprising $100 \%$ sensitivity and $100 \%$ specificity for multiple sclerosis.

Patients with this venous insufficiency underwent catheter-based venography, during which stenoses were treated with balloon angioplasty. This technique has been provocatively dubbed the "liberation procedure." In an open-label trial of the technique, Zamboni and colleagues saw an improvement in measures of inflammation in cases of early, relapsing-remitting multiple sclerosis, but no significant changes in disability or quality of life was seen among patients with later stages of the disease. ${ }^{5}$ Despite the reported lack of efficacy in cases of progressive multiple sclerosis, stories of dramatic improvements following the liberation procedure among patients with this form of the disease spread quickly. Social networks and news programs chronicled how patients showed immediate improvements in sensation, strength and coordination after surgery. Some patients who had been using wheelchairs for years were now walking without assistance. Websites promoted medical tourism, and grassroots advocacy organizations pushed for widespread availability of the procedure. Perhaps for no other condition has social media been as effective in promoting a medical theory as with chronic cerebrospinal venous insufficiency.

Many scientists and clinicians involved in researching and treating multiple sclerosis have been skeptical of this "new" theory, citing a lack

All editorial matter in CMAJ represents the opinions of the authors and not necessarily those of the Canadian Medical Association. 
of objective evidence. These same scientists and clinicians were accused by proponents of the theory of territorialism or being beholden to the pharmaceutical companies that profit from the current treatments for the disease. Health agencies and foundations for multiple sclerosis were caught in the middle - pushed by patients to wholly embrace the theory and chastised by doubtful scientists and clinicians that any money spent on supporting this theory would be money lost from finding the real cure.

The liberation procedure is associated with real risks, with reports of death and other lifethreatening complications related to the treatment. The only published studies of the liberation procedure are uncontrolled, open-label trials. Perhaps most importantly, the very existence of chronic cerebrospinal venous insufficiency and its association with multiple sclerosis remain unclear. The original validation studies compared the results of ultrasonography with diagnoses of multiple sclerosis, but there was no independent validating vascular measure. The technical criteria for reflux and stenosis were adopted from other vascular systems, with uncertain relevance to the jugular, vertebral and intracranial veins. Independent confirmation studies were needed, but the subsequent studies from different groups came to different conclusions.

In a meta-analysis commissioned by the Canadian Institutes of Health Research, Laupacis and colleagues looked at the eight studies that have been published evaluating the association between chronic cerebrospinal venous insufficiency and multiple sclerosis. ${ }^{6}$ These studies examined a total of 664 patients with multiple sclerosis and an equal number of controls (528 healthy controls, and 136 controls with neurologic diseases other than multiple sclerosis). The authors found that chronic cerebrospinal venous insufficiency was more frequent among people with multiple sclerosis than among healthy controls (odds ratio 13.5, 95\% confidence interval 2.6-71.4). Even the most conservative sensitivity analysis found a significant association between chronic cerebrospinal venous insufficiency and multiple sclerosis.

Despite the results of this meta-analysis, we are still far from proving that chronic cerebrospinal venous insufficiency is the cause of multiple sclerosis and embracing the liberation procedure. There was substantial heterogeneity between the studies analyzed by Laupacis and colleagues in terms of the frequency of chronic cerebrospinal venous insufficiency and the strength of the associations seen. The simple prevalence of chronic cerebrospinal venous insufficiency among patients with multiple scle- rosis varied from $0 \%$ to $100 \%$, which suggests that the performance of ultrasonography to determine the presence of chronic cerebrospinal venous insufficiency varied greatly between the studies. Potential biases from patient unblinding may also have affected the results. The comparison between multiple sclerosis and other neurologic diseases found only a trend in association, raising the question of whether chronic cerebrospinal venous insufficiency may be a common finding in a variety of neurologic disorders.

The meta-analysis of Laupacis and colleagues cannot differentiate between causal and noncausal associations. Indeed, findings of chronic cerebrospinal venous insufficiency during ultrasonography may simply be a result of hydration, with mild dehydration being preferred by patients with multiple sclerosis who have neurogenic bladders.

Additional independent studies to confirm the association between chronic cerebrospinal venous insufficiency and multiple sclerosis are under way. However, ultrasonographic assessment of chronic cerebrospinal venous insufficiency is not straightforward, and the standardization of protocols and the use of experienced ultrasonographers are important. In June 2011, Health Canada announced that planning for a phase I/II clinical trial was in progress. The trial is expected to focus on assessing outcomes and the safety of venoplasty for patients with multiple sclerosis. The metaanalysis by Laupacis and colleagues is a good starting point; however, much work remains to be done before we can be certain whether chronic cerebrospinal venous insufficiency is a paradigm shift in the classic sense, as conceived by Thomas Samuel Kuhn, ${ }^{7}$ or just another fad.

\section{References}

1. Cruveilhier J. Pathological anatomy of the human body. Paris (France): Billière; 1829.

2. Charcot JM. Lectures on diseases of the nervous system. London (UK): The New Sydenham Society; 1877.

3. Zamboni P, Menegatti E, Bartolomei I, et al. Intracranial venous haemodynamics in multiple sclerosis. Curr Neurovasc Res 2007; 4:252-8.

4. Zamboni P, Galeotti R, Menegatti E, et al. Chronic cerebrospinal venous insufficiency in patients with multiple sclerosis. J Neurol Neurosurg Psychiatry 2009;80:392-9.

5. Zamboni P, Galeotti R, Menegatti E, et al. A prospective openlabel study of endovascular treatment of chronic cerebrospinal venous insufficiency. J Vasc Surg 2009;50:1348-58.e1-3.

6. Laupacis A, Lillie E, Dueck A, et al. Association between chronic cerebrospinal venous insufficiency and multiple sclerosis: a meta-analysis. CMAJ 2011;183:E1203-12.

7. Kuhn, TS. The structure of scientific revolutions. Chicago (IL): University of Chicago Press; 1962.

Affiliations: Robert Fox is with the Mellen Center for Multiple Sclerosis Treatment and Research, Department of Neurology, Neurological Institute, and the Cleveland Clinic, Lerner College of Medicine, Cleveland Clinic, Cleveland, Ohio.

Acknowledgement: The author thanks Dr. Alex Rae-Grant for discussion and review of this manuscript. 